\title{
High Spatial Resolution Spectroscopy in a FE-SEM: X-ray Microanalysis and Electron Energy-Loss Spectroscopy
}

\author{
Hendrix Demers ${ }^{1}$, Nicolas Brodusch ${ }^{1}$, and Raynald Gauvin ${ }^{1}$ \\ 1. Department of Mining and Materials Engineering, McGill University, Montreal, Quebec, Canada.
}

For developing new technologies, it is important to characterize materials at the nanoscale. To achieve high resolution, field emission scanning electron microscopes (FE-SEM) with low voltage scanning transmitted electron microscope (STEM) were developed. Furthermore, some instrument has diffraction analysis, electron energy loss spectrometry (EELS), and energy dispersive spectrometry (EDS) systems, which allow crystallographic and chemical characterization at the nanoscale materials. However, the scanning electron microscope (SEM) was primary developed for imaging applications. With the introduction of the $\mathrm{Si}(\mathrm{Li}) \mathrm{EDS}$, simultaneous imaging and $\mathrm{x}$-ray microanalysis became possible. However, long working distance and high current were needed because the position and small solid angle of the EDS detector. SEM was initially and is still optimized for imaging applications, where the high spatial resolution is generally obtained at short working distance. This problem is still relevant today and unfortunately x-ray microanalysis is never performed in the best imaging conditions, i.e., not with the smallest probe size. With the introduction of improved geometry and design, modern silicon drift detector (SDD) systems (like the annular detector or low profile windowless detector) can be used for high spatial resolution x-ray microanalysis.

Figure 1 show an example of high spatial resolution x-ray microanalysis. The map was acquired at low accelerating voltage to reduce the $\mathrm{x}$-ray emission interaction volume. The spatial resolution of the Au and $\mathrm{C}$ x-ray maps were similar than the one calculated on the secondary electron micrograph. Recent development in $x$-ray detection technology like the windowless EDS SDD and the soft $x$-ray emission spectrometer (SXES) allow the measurement of soft x-ray lines $(>50 \mathrm{eV})$, which permits the detection of most elements of the periodic table at lower accelerating voltage. Another approach is to use thin film sample and do the x-ray microanalysis in the low accelerating voltage STEM mode. Figure 2 show an example of this approach. X-ray map of a thin film of an Al-Li alloy $(\sim 80 \mathrm{~nm})$ was obtained and the Copper rich $\mathrm{T}_{1}\left(\mathrm{Al}_{2} \mathrm{CuLi}\right)$ plates were clearly observed. The plates are 1 to $2 \mathrm{~nm}$ wide. Furthermore, the low concentration of $\mathrm{Zn}$ and the decrease of $\mathrm{Al}$ concentration in the $\mathrm{T}_{1}$ plates versus the $\mathrm{Al}$ matrix was also observed in an high resolution X-ray line scan (not shown).

On our FE-SEM (Hitachi SU-9000), the energy dispersive spectrometry (EDS) is complemented by an electron energy loss spectrometry (EELS) analytical system. Examples of low voltage EELS were previously presented to validate the possibility of low voltage EELS [1,2]. We are currently working to acquire EELS and EDS data simultaneously to clearly observe the complete composition of each Al-Li alloy phase as it is not possible to detect the low concentration of $\mathrm{Zn}$ with EELS and the Li detection with windowless EDS is challenging. These examples show that after 50 years of development of x-ray microanalysis, we are starting the era of $\mathbf{x}$-ray nanoanalysis with low voltage SEM/STEM instrument. 


\section{References:}

[1] T. Sunaoshi et al, Microscopy and Microanalysis 22 (2016), pp. 604-605.

[2] Y. Yamazawa et al, Microscopy and Microanalysis 22 (2016), pp. 50-51.

[3] D.C. Joy, Journal of Microscopy, 208 (2002), pp. 24-34.

[4] J. Kim et al, Journal of Vacuum Science Technology B, 25 (2007), pp. 1771-1775.

[5] This work was supported by Hitachi High-Technologies Canada Inc.
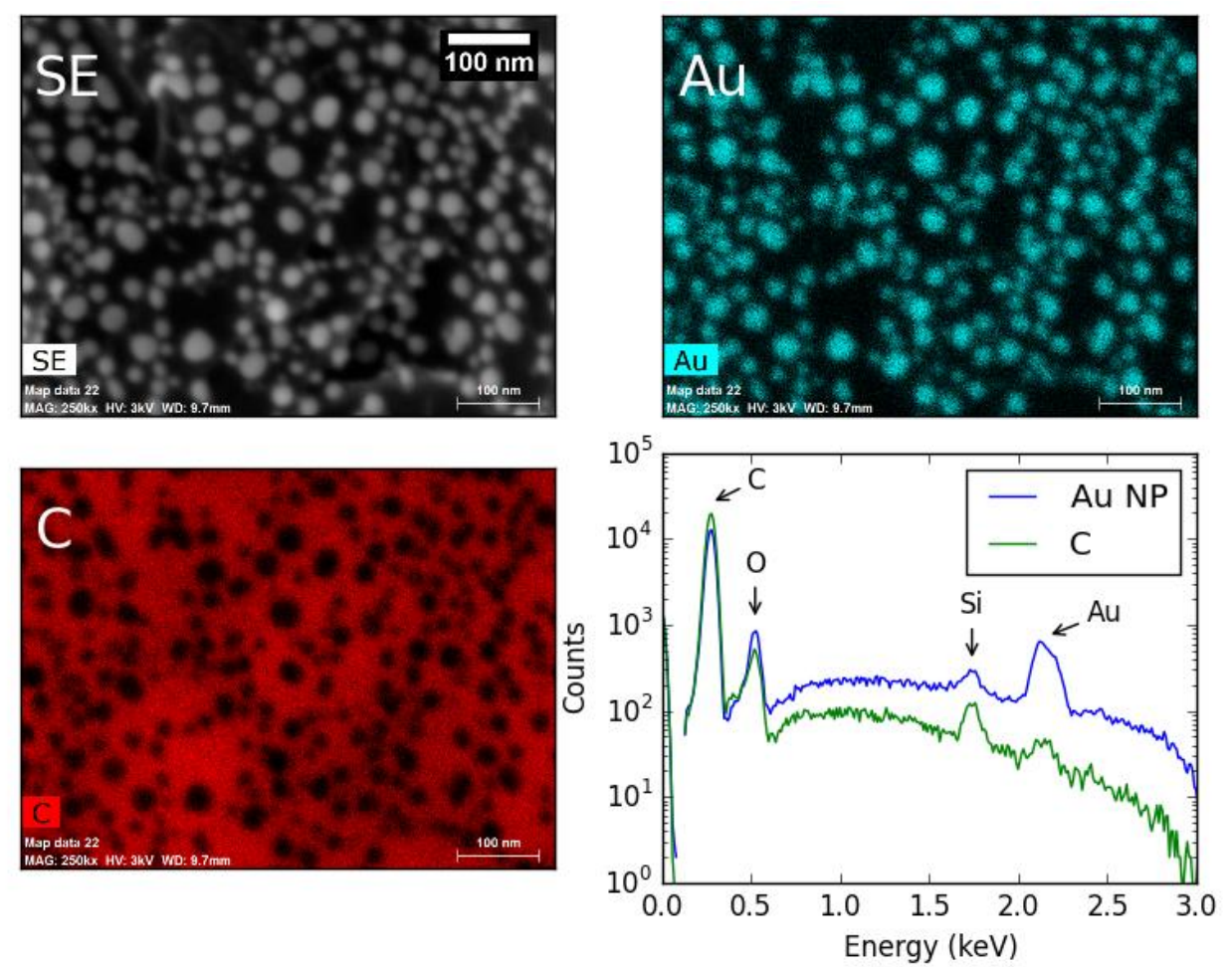

Figure 1. An example of high resolution $\mathrm{x}$-ray microanalysis at low accelerating voltage $(3 \mathrm{kV}) \mathrm{was}$ acquired for Gold nanoparticles on Carbon substrate. A resolution of $7.3 \mathrm{~nm}, 8.6 \mathrm{~nm}$, and $9.4 \mathrm{~nm}$ were measured with SMART-J [3-4] on the SE micrograph, the Au X-ray map, and the C x-ray map, respectively.
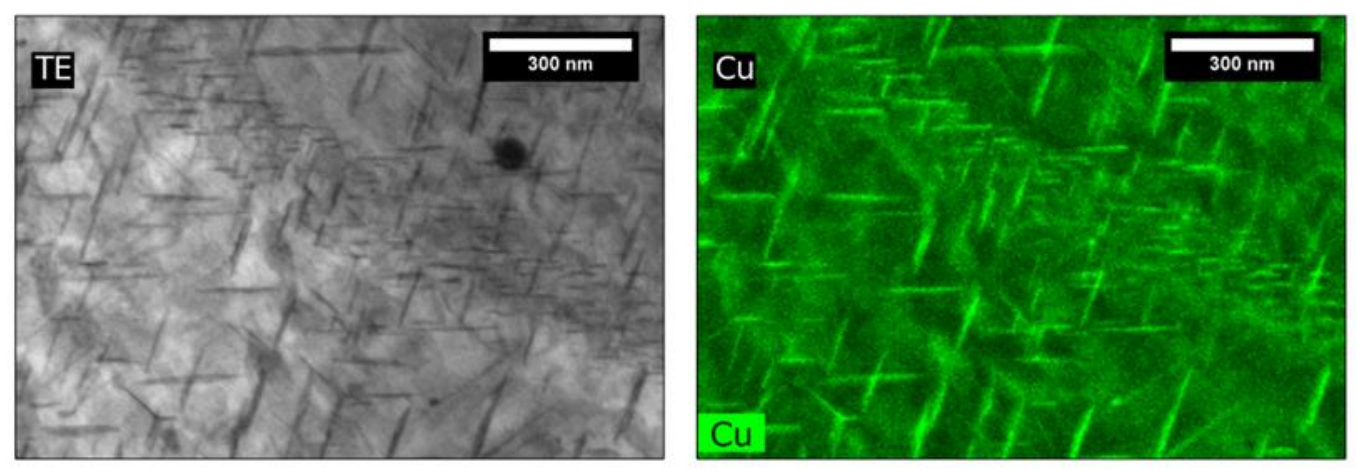

Figure 2. High spatial resolution $x$-ray elemental map of an Al-Li 2099 alloy at $20 \mathrm{kV}$ in SEM/STEM mode. The $\mathrm{T}_{1}\left(\mathrm{Al}_{2} \mathrm{CuLi}\right)$ plates observed in the bright-field micrograph (TE) are clearly visible in the $\mathrm{Cu}$ map. 